\title{
Gallium Ga 68 Pentixafor
}

National Cancer Institute

\section{Source}

National Cancer Institute. Gallium Ga 68 Pentixafor. NCI Thesaurus. Code C141632.

A radioconjug ate composed of a synthetic, cyclic pentapeptide analog of stromal-cell derived factor-1 (SDF-1 or CXCL12), which is a lig and for chemokine receptor C-X-C chemokine receptor type 4 (CXCR4), that is radiolabeled, via the macrocyclic chelating agent dodecanetetraacetic acid (DOTA), with the radioisotope gallium Ga 68 (Ga68; 68Ga), with potential use for imaging CXCR4-expressing cells upon positron emission tomography (PET)/computed tomography (CT). Upon administration of 68Ga pentixafor, the pentixafor moiety targets and binds to CXCR4-expressing cancer cells. Upon PET/CT, CXCR4-expressing cancer cells can be visualized and the expression status of the receptor can be assessed. CXCR4, a marker of poorly differentiated cells, is overexpressed on various cancer cells, and plays a key role in tumor growth, progression, invasiveness and metastasis. 